\title{
Variaciones de la infracomunidad parásita durante la ontogenia de Chromis cyanea (Perciformes: Pomacentridae) en la costa norte de La Habana, Cuba
}

\author{
Ramón Alexis Fernández Osorio ${ }^{1}$, Raúl Igor Corrada Wong ${ }^{1}$ \& Maickel Armenteros ${ }^{2}$ \\ 1. Acuario Nacional de Cuba, Avenida 1ra y Calle 60, Playa, La Habana, Cuba; \\ alexisf@acuarionacional.cu, raulc@acuarionacional.cu \\ 2. Centro de Investigaciones Marinas, Universidad de la Habana, Calle 16 y 3ra, Playa, La Habana, Cuba; \\ maickel@cim.uh.cu
}

\author{
Recibido 14-VIII-2014. C Corregido 01-II-2015. Aceptado 09-III-2015.
}

\begin{abstract}
Variations of the parasitic infracommunity during the ontogeny of Chromis cyanea (Perciformes: Pomacentridae) in the North coast of Havana, Cuba. The infracommunity of parasites of Chromis cyanea (Pisces: Pomacentridae) was studied along the ontogenetic development in the North coast of Havana, Cuba. The objectives were: a- to prove that the core species appears before the strange and stochastic species and they are responsible for the structure in the infracommunity, b- to determine if there is a relationship among the ecological describers of the parasitic infracommunity with the total length. A total of 278 specimens of $C$. cyanea were captured during the dry season (April) of 2010. The body size range was from 1.5 to $11.5 \mathrm{~cm}$ including juvenile and adults. We collected 2861 parasite specimens belonging to 20 taxa: Crustacea (5), Nematoda (5), Trematoda (4), Cestoda (2), Monogenea (2), Turbellaria (1) and Ciliophora (1). The taxa Tetraphyllidea and Anilocra chromis constituted the core of the parasitic infracommunity. The sequence of appearance and persistence of these taxa during the ontogenetic development, supported the hypothesis of the core species. The changes in the infracommunity, from $6 \mathrm{~cm}$ body size, could be the result of an accumulative effect combined with changes of the diet that caused the ingestion of new parasite infective stages. We concluded that the ontogenetic development of $C$. cyanea constitutes an important variable in the formation of the parasitic infracommunity. Rev. Biol. Trop. 63 (3): 717-726. Epub 2015 September 01.
\end{abstract}

Key words: Chromis cyanea, infracommunity, ontogeny, parasites, marine fishes, core species, Cuba.

La infracomunidad es el conjunto de parásitos de diferentes especies que habitan en un individuo hospedero y constituye una unidad de estudio espacialmente discontinua en la que cada individuo hospedero es considerado una muestra aleatoria del hábitat (Holmes \& Price, 1986; Bush, Lafferty, Lotz, \& Shostak, 1997). Esto se debe a que hospederos de la misma especie adquieren parásitos de un conjunto local a partir de la ingestión de dietas similares $\mathrm{y}$ estar sometidos a similares condiciones y factores del hábitat.

El proceso de colonización de los parásitos a las infracomunidades puede ser secuencial si hay un patrón ontogenético de variación en la dieta de los hospederos. Esto puede modificar radicalmente la composición y abundancia de las especies de parásitos en hospederos que cohabitan (Díaz \& George-Nascimento, 2002; Muñoz \& Delorme, 2011). Por consiguiente, el tamaño de los hospederos se puede tomar como indicador de edad y composición de las infracomunidades; para encontrar evidencia de dichas variaciones ontogenéticas se requiere que el espectro de edad de los peces muestreados sea lo suficientemente amplio como para que sean detectables los cambios (Rodríguez \& George-Nascimento, 1996). Sin embargo, son escasos los estudios a nivel de población de hospederos en peces marinos, que abarquen 
todo el rango de edades o tallas, para así precisar el papel de la edad del hospedero en el proceso de colonización de la infracomunidad.

El cambio ontogénico de la dieta y/o hábitat por parte del huésped es un mecanismo que puede dar lugar a un patrón de anidamiento en la estructura de las infracomunidades (Poulin \&Valtonen, 2001; Vidal-Martínez \& Poulin, 2003). No obstante, la adición de especies parásitas a una infracomunidad base formada por especies que no se ven afectadas por la talla del hospedero, podría crear también una estructura anidada, sin ocurrir un cambio estricto en la dieta (Zelmer \& Arai, 2004). Si es importante identificar los patrones en la estructura de las comunidades parásitas, y detectar los procesos que los crean. Los procesos epidemiológicos, o capacidad de colonización/extinción de las especies parásitas, han sido sugeridos como causas de la estructura de ciertas comunidades parásitas (Morand, Rohde, \& Hayward, 2002). Para explicar los patrones de distribución de las especies, Hanski (1982) planteó la hipótesis de especies núcleo (las cuales se encontrarán en muchas localidades en abundancia alta) y de especies raras (las cuales se encontrarán distribuidas en pocas localidades en abundancia baja).

La hipótesis de que el incremento del nivel de infección por parásitos aumenta con la edad del hospedero fue esbozada por Dogiel, Petrushevski and Polyanski (1958) y fue actualizada por Labropoulou, Machias, Tsimenides and Eleftheriou (1997). La hipótesis plantea que los cambios radicales en los niveles de parasitación están supeditados a cambios estrictos en la dieta a partir de la talla de maduración y diferenciación sexual. Pero actualmente existen evidencias empíricas que sugieren la influencia de otros factores ecológicos del hospedero como la talla y edad (Johnson, Nelson, \& Dock, 2004), el tipo de hábitat (Muñoz, Grutter, \& Cribb, 2006), la movilidad (Kennedy, 1990), y el comportamiento social y posible formación de cardúmenes (Luque, Mouillot, \& Poulin, 2004). La exploración de estos factores (dieta, talla) en un contexto de análisis de la infracomunidad a lo largo de la ontogenia puede indicar los procesos claves que determinan la formación de tales comunidades y su influencia sobre el hospedero.

Chromis cyanea (Poey 1860) es una de las especies marinas más comunes y abundantes de los arrecifes cubanos (Claro, Lindeman, \& Parenti, 2001). La especie tiene un alto valor ornamental pero hasta la fecha no existen estudios que aborden el desarrollo de la infracomunidad parásita a lo largo de su desarrollo ontogenético. Por este motivo nos proponemos como objetivos: (i) probar la hipótesis que las especies núcleo son responsables de la estructura en la infracomunidad y que por tanto aparecen antes que las especies raras y estocásticas; y (ii) determinar si existe relación de los descriptores de la infracomunidad parásita con el desarrollo ontogenético de $C$. cyanea inferido a partir de la longitud total.

\section{MATERIALES Y MÉTODOS}

Área de estudio y recolecta: El estudio se realizó en La Habana, costa noroccidental de Cuba, en las coordenadas $\left(23^{\circ} 07^{\prime} 06.1^{\prime \prime}\right.$ $\left.\mathrm{N}-082^{\circ} 26^{\prime} 56.4^{\prime \prime} \mathrm{W}\right)$. Las colectas se realizaron durante el mes de abril del 2010 (estación de seca) con el objetivo de limitar el posible efecto de las variaciones estacionales en la infracomunidad parásita. Los ejemplares de $C$. cyanea fueron capturados procurando cubrir el máximo intervalo de talla descrito en la ontogenia de la especie; ello indica un valor de talla máxima de $12.5 \mathrm{~cm}$ según Böhlke y Chaplin (1968). La captura se realizó mediante buceo autónomo, con el auxilio de una red de 0.3 $\mathrm{mm}$ de abertura, con un marco cuadrado de 40 $\mathrm{cm}$. Los ejemplares capturados se colocaron en nailon de polietileno transparente para ser trasladados hasta el área de cuarentena del Acuario Nacional de Cuba.

Trabajo de laboratorio: Para evaluar la infracomunidad a lo largo de la ontogenia, los peces fueron divididos en clases de talla según dos criterios: (1) Se dividieron los peces en diez clases de talla de $1 \mathrm{~cm}$ de largo total (LT) según Sturges (1926); y (2) Se dividieron los 
peces en dos clases de talla, menores y mayores de $6 \mathrm{~cm}$ de LT. Los peces fueron sacrificados mediante un corte en la región cervical (Fernández \& Corrada, 2009). Se midió la LT con un pie de rey de $0.1 \mathrm{~mm}$ de precisión y el peso en una balanza técnica de $0.1 \mathrm{~g}$ de precisión. Los procedimientos parasitológicos aplicados fueron los descritos por Moravec et al., (1995). La identificación de los parásitos colectados se basó en: Yamaguti (1963) para monogéneos; Vidal-Martínez, Aguirre-Macedo, Tomás, David y Edgar (2001) para tremátodos, nemátodos y céstodos; Noga (1996) para turbelarios, y Kensley y Schotte (1989), y Cressey (1991) para crustáceos.

La magnitud de las parasitosis en los especímenes de C. cyanea fue descrita por tres variables (Bush et al., 1997): (i) prevalencia: porcentaje de individuos hospederos parasitados; (ii) abundancia: media de individuos parásitos por hospedero parasitado o no parasitado; e (iii) intensidad media: media de individuos parásitos por hospedero parasitado. El análisis de la infracomunidad consideró la riqueza como el número de taxa parásitos, la abundancia total como el número de individuos parásitos en cada infracomunidad (Holmes \& Price, 1986) y la diversidad mediante el índice de Brillouin (Magurran, 1988) que se recomienda para comunidades completamente censadas.

Se aplicó la prueba no paramétrica de Kruskal-Wallis para detectar diferencias entre clases de talla. Para evaluar la existencia o no de patrones ontogenéticos en las infracomunidades a lo largo de las 10 clases de talla, se realizó un análisis de regresión simple. Las variables dependientes fueron la riqueza, abundancia total, diversidad y la composición de la infracomunidad, en tanto que el largo total fue la variable independiente. La composición de las infracomunidades fue evaluada como un vector de abundancia relativa expresada como porcentaje del total de individuos parásitos que cada taxón aporta en cada infracomunidad. Se realizó un análisis de correspondencia con efecto de arco corregido (DCA) que permitió ordenar las infracomunidades en una dimensión. La composición de las infracomunidades se representa a través de los puntajes de los taxa en el primer eje de ordenación, cuyas unidades son desviaciones estándar $(x 100)$ de la tasa de reemplazo a lo largo del gradiente. Para realizar el DCA solo se consideraron los taxa con más de cinco ocurrencias en el total de cada intervalo, debido a que este análisis es sensible a las especies raras (Jongman, Braak, \& Tongeren, 1995).

Para establecer las posibles diferencias de la infracomunidad, antes y después de la talla de seis centímetros de largo total, se evaluó la riqueza, abundancia, y los índices de equitatividad de Pielou, de diversidad de Brillouin y de dominancia numérica de Berger-Parker (Magurran, 1988). Las comparaciones se hicieron mediante la prueba U de Mann-Whitney (Jongman et al., 1995). Los ejemplares colectados e identificados, fueron depositados en las Colecciones Científicas del Acuario Nacional de Cuba.

\section{RESULTADOS}

Análisis parasitológico: Se examinaron 278 ejemplares de $C$. cyanea y el largo total fluctuó entre 1.5 y $11.5 \mathrm{~cm}$. Se obtuvo un total de 2861 parásitos pertenecientes a 20 taxa distribuidos en siete clases. Crustacea y Nematoda fueron los mejores representados con cinco taxa cada uno, Trematoda con cuatro, Cestoda y Monogenea con dos, y Turbellaria y Ciliophora con uno. De los taxa colectados, seis se encontraron en estadío larval y 14 en fase adulto.

El $60 \%$ del total de hospederos presentó algún taxón parásito y en cada clase de talla el $53 \%$ o más estuvo parasitado por al menos un taxón. E1 85\% de las especies parásitas mostraron prevalencia por debajo del $20 \%$. Más del $60 \%$ de las especies mostraron una intensidad de parasitación por debajo de cinco individuos por hospedero (Cuadro 1). La entidad Tetraphyllidea mostró la mayor prevalencia en todas las clases de talla con un rango de valores entre 20 y $52 \%$. Le siguen en orden de prevalencia Anilocra chromis, Eutetrarhynchus sp. y Caligus ocyurus. Tetraphyllidea tuvo los mayores 


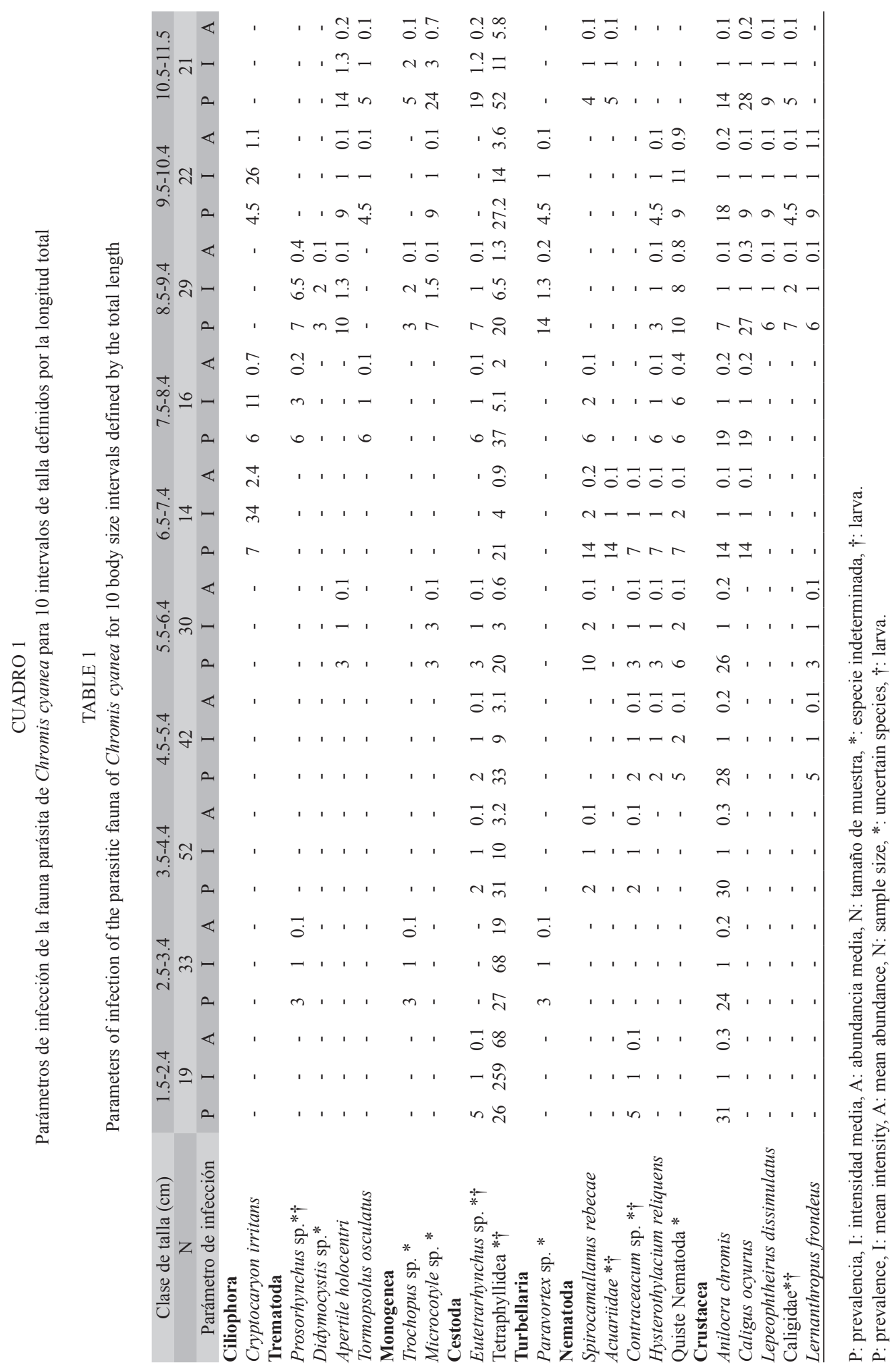


valores de intensidad promedio con un máximo de 260 individuos en el primer intervalo de talla (Cuadro 1).

El taxón más prevalente y abundante fue Tetraphyllidea que no presentó diferencias estadísticas significativas entre intervalos de tallas para la abundancia $\left(\mathrm{H}_{(9 ; 278)}=11.61, \mathrm{p}=\right.$ $0.23)$ y la intensidad media $\left(\mathrm{H}_{(9 ; 82)}=13.02, \mathrm{p}\right.$ $=0.16$ ). Anilocra chromis no presentó diferencias estadísticas significativas en la abundancia $\left(\mathrm{H}_{(9 ; 278)}=9.71, \mathrm{p}=0.37\right)$ ni en la intensidad promedio $\left(\mathrm{H}_{(9 ; 65)}=4, \mathrm{p}=0.91\right)$. El taxón dominante fue Tetraphyllidea en cada intervalo de talla, con un valor máximo de $0.42 \pm 0.43$.
Análisis ontogenético: El análisis de regresión entre el LT y los descriptores de la infracomunidad, indicó una relación lineal pobre aunque la pendiente de la regresión para cada uno de los descriptores fue similar y estadísticamente significativa (Fig. 1A, Fig. 1B, Fig. 1C). La posición en el gradiente de composición de los taxa más abundantes se indica en la figura 1D. La correspondencia en el primer eje del DCA de los taxa parásitos mostró un valor de 0.67 y la composición de la infracomunidad presentó un ligero incremento con el desarrollo ontogenético de $C$. cyanea.
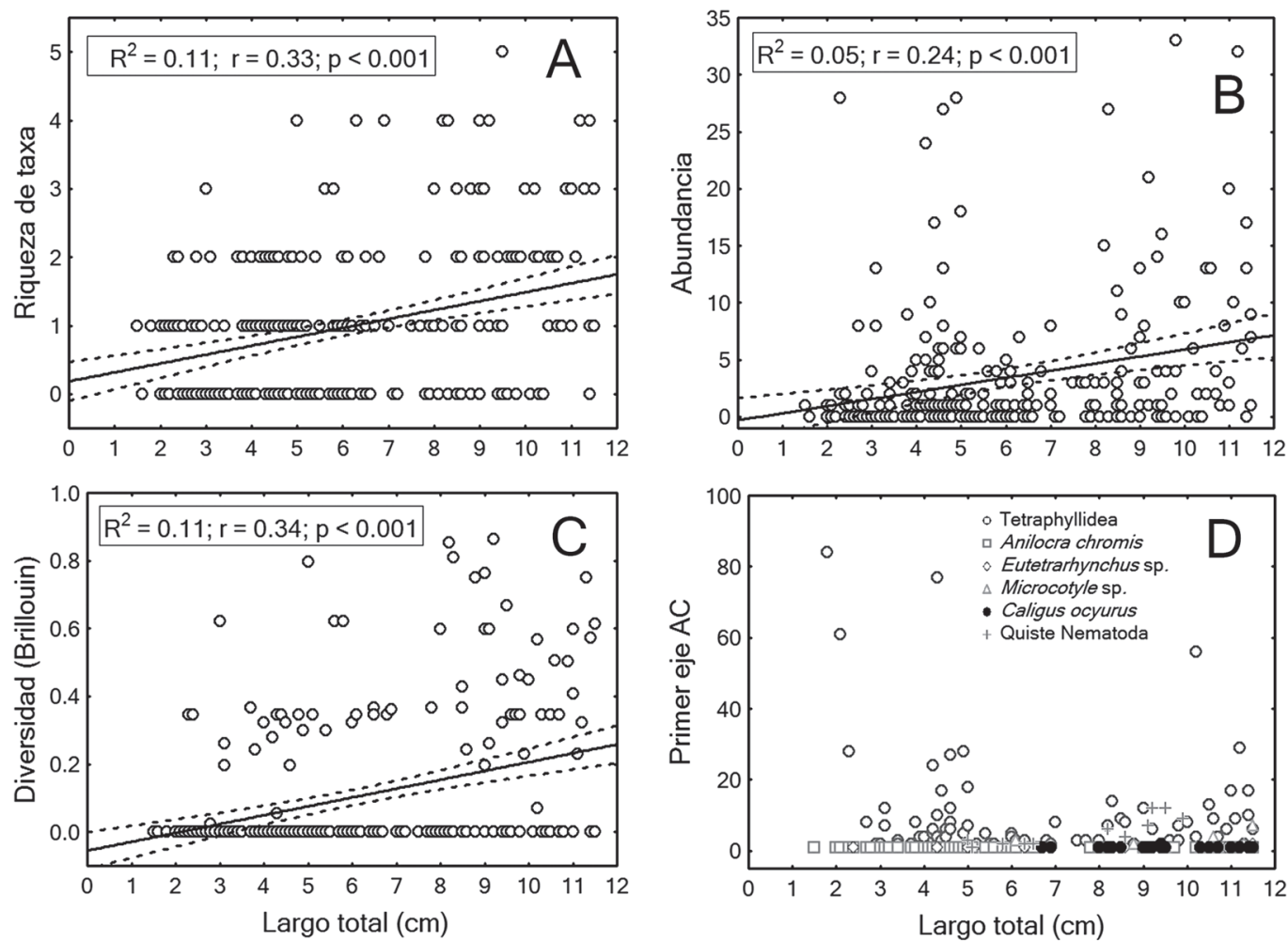

Fig. 1. Asociación de descriptores de la infracomunidad parásita con la longitud de 278 especímenes de Chromis cyanea. A) Riqueza de taxa parásitos; B) Abundancia; C) Índice de diversidad de Brillouin; y D) Composición de la comunidad medida por los puntajes del primer eje de un análisis de correspondencia (AC). Se indican los valores del coeficiente de determinación $\left(\mathrm{R}^{2}\right)$, coeficiente de correlación ( $\mathrm{r}$ ) y la probabilidad (p) de cometer error de tipo I en un análisis de regresión. Fig. 1. Association of descriptors of the parasitic infracommunity with the length of 278 specimens of Chromis cyanea. A) Richness of parasitic taxa; B) Abundance; C) Index of diversity of Brillouin; and D) Composition of the community as measure by the scores of the first axis of the correspondence analysis. The values of the coefficient of determination are indicated $\left(\mathrm{R}^{2}\right)$, coefficient of correlation $(\mathrm{r})$; and the probability (p) to make type I error in an analysis of regression. 
(Fig. 1D). Los taxa Tetraphyllidea y A. chromis parasitan al hospedero a partir de la talla de $1.5 \mathrm{~cm}$ de LT y persisten durante toda la ontogenia (Fig. 1D).

El análisis realizado de la composición de especies antes y después de la talla de maduración sexual de C. cyanea, reveló un incremento notable de los índices cuantitativos del parasitismo, para la mayoría de los taxa hacia la segunda clase de talla. La composición por grupos taxonómicos se comportó de forma heterogénea dentro de ambos intervalos. El número de entidades parásitas incrementó en la segunda clase de talla. Tetraphyllidea disminuyó la intensidad y la abundancia en el segundo intervalo, aunque incrementó ligeramente la prevalencia (Cuadro 2).

Los descriptores de la infracomunidad mostraron un comportamiento diferente antes y después de la talla de $6 \mathrm{~cm}$ de LT. El promedio de especies por hospedero fue de $0.65 \pm 0.78$ para la clase $<6 \mathrm{~cm}$ y $1.32 \pm 1.24$ para la clase $>6 \mathrm{~cm}$. El promedio de parásitos por hospedero resultó mayor en la primera clase de talla (14.0 $\pm 93.2)$ y menor que en la segunda (5.0 \pm 8.5$)$; esto se debe a intensidades de parasitación extremas por Tetraphyllidae que oscilaron entre 3 y 260 parásitos. La diversidad de Brillouin mostró un incremento notable en los especímenes $>6 \mathrm{~cm}$ de LT (Cuadro 3).

\section{CUADRO 2}

Parámetros de infección de la fauna parásita de Chromis cyanea para dos intervalos de talla definidos por la longitud total: $<6 \mathrm{~cm}(\mathrm{n}=165)$ y $>6 \mathrm{~cm}(\mathrm{n}=113)$

\section{TABLE 2}

Parameters of infection of the parasitic fauna of Chromis cyanea for two body size intervals defined by the total length

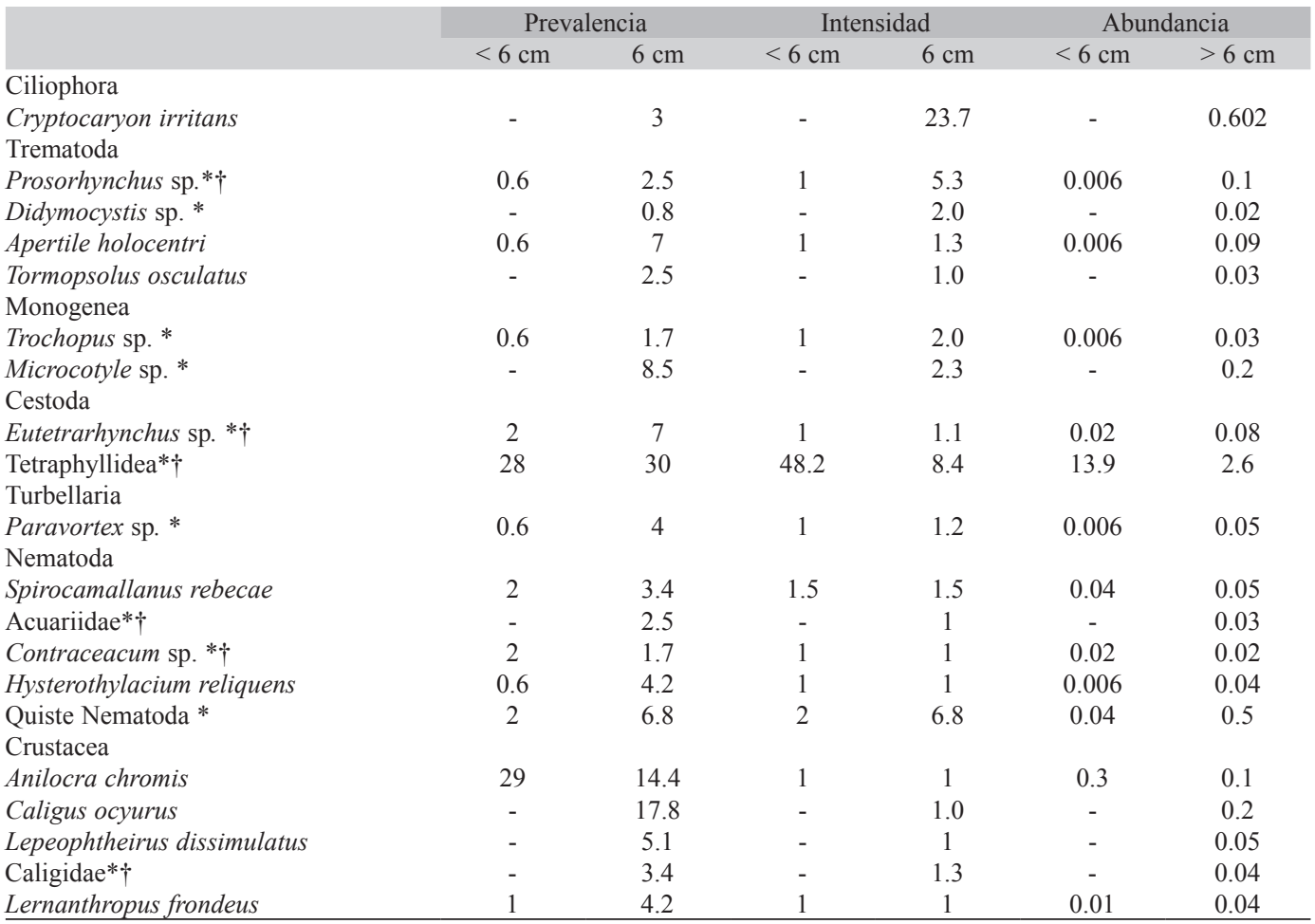

*: especie indeterminada (uncertain species), $\uparrow$ : larva (larvae) 
Descriptores ecológicos (promedio \pm desviación estándar) de la fauna parásita de Chromis cyanea para dos intervalos de talla definidos por la longitud total (LT): $<6 \mathrm{~cm}(\mathrm{n}=165)$ y $>6 \mathrm{~cm}(\mathrm{n}=113)$

TABLE 3

Ecological descriptors (average \pm standard deviation) of the parasitic fauna of Chromis cyanea for two body size intervals defined by the total length

\begin{tabular}{lcc}
\multicolumn{1}{c}{ Descriptor } & $\mathrm{LT}<6 \mathrm{~cm}$ & $\mathrm{LT}>6 \mathrm{~cm}$ \\
Especies por hospederos* & $0.65 \pm 0.78$ & $1.32 \pm 1.24$ \\
Parásitos por hospederos* & $14 \pm 93$ & $5 \pm 9$ \\
Diversidad de Brillouin* & $0.04 \pm 0.13$ & $0.17 \pm 0.25$ \\
Equidad de Pielou* & $0.09 \pm 0.26$ & $0.28 \pm 0.9$ \\
Dominancia numérica de Berger-Parker & $0.25 \pm 0.41$ & $0.22 \pm 0.36$ \\
Taxón dominante & Tetraphyllidae & Tetraphyllidae \\
\hline
\end{tabular}

* indica diferencia significativa según una prueba U de Mann-Whitney.

\section{DISCUSIÓN}

Este estudio es el primero en caracterizar la fauna parásita de Chromis cyanea a lo largo de la ontogenia de la especie. Otros estudios (Fernández \& Corrada, 2009) han analizado la infracomunidad de esta especie hospedera, pero solo en estadíos adultos.

La mayor parte de los parásitos de Chromis cyanea son especies generalistas que se transmiten por mediación de otras especies de peces simpátricas. El 35\% de las especies parásitas son especialistas y solo la entidad quiste de nemátodo se definió con estatus desconocido. Resultados similares en el predominio de larvas de céstodos en C. cyanea fueron obtenidos por Fernández y Corrada (2009). Otros estudios realizados en peces marinos han descrito estos parásitos con altos índices de infección (Sánchez-Ramírez \& Vidal-Martínez, 2002; Pérez de Olmo, 2008; Muñoz \& Delorme, 2011). Gonzáles y Vidal-Martínez (2008) caracterizaron las comunidades parásitas de Symphurus plagiusa y detectaron una larva Trypanorhyncha con alta intensidad de parasitación y prevalencia. La presencia de larvas con valor de prevalencia elevado puede ser explicada por los hábitos zooplanctófagos de $C$. cyanea que permiten incorporar por vía trófica especies de crustáceos que constituyen hospederos intermediarios en el ciclo de vida de los céstodos.
La especie generalista Anilocra chromis infestó a Chromis cyanea desde las primeras clases de talla pero siempre con una intensidad de un individuo. Fernández y Corrada (2009) señalan que este parásito siempre se fija en la parte superior del opérculo y que luego de la infestación, se podrían activar mecanismos de exclusión que unidos a la limitada disponibilidad del sitio de fijación impidan la entrada a otros individuos de la misma especie. Tetraphyllidea también parasitó a $C$. cyanea desde las primeras clases de talla y persistió durante todo desarrollo ontogenético. Esto puede explicarse por una elevada tasa de residencia que se favorece por la dieta de C. cyanea; constituida fundamentalmente por copépodos, quetognatos, anfípodos, moluscos y poliquetos que pueden ser portadores de nuevos estadíos infectivos (Pastor \& Báez-Hidalgo, 2003).

La longitud total del hospedero, como indicador de la ontogenia, tiene un efecto débil sobre la infracomunidad parásita. Pero ha resultado una variable relevante para explicar las variaciones en la infracomunidad parásita de otras especies de peces marinos (Iannacone \& Alvariño, 2009; Muñoz \& Delorme, 2011; Muñoz \& Zamora, 2011). Sin embargo, en estudios realizados en las familias de peces Serranidae y Sciaenidae la talla es irrelevante. Esta ausencia de efecto de la talla podría deberse a dos aspectos: (i) el espectro de tallas analizado en los otros estudios ha sido 
reducido y/o (ii) los hospederos durante su ontogenia no migran para ocupar otros hábitats (Oliva \& Luque, 2002; Iannacone, Morón, \& Guizado, 2010).

El incremento en la riqueza y abundancia de parásitos ocurre en los individuos de mayor talla. Esto podría deberse al efecto combinado de: (i) la acumulación de parásitos a lo largo de la ontogenia; (ii) una tasa mayor de ingesta o (iii) la ingesta de presas mayores más parasitadas. La conducta alimentaria de C. cyanea incluye migraciones diarias que permiten explorar nuevos hábitats con mayor disponibilidad de alimento dentro del arrecife (Claro et al., 2001). Esto facilita la incorporación de parásitos por vía trófica que afectan la composición y abundancia de la infracomunidad (Luque \& Poulin, 2007). Adicionalmente, las variaciones en la composición de la infracomunidad con la talla pueden ser resultado del incremento en la disponibilidad y tamaño de los nichos que ofrecen una mayor heterogeneidad del hábitat y mayor superficie en los órganos (Luque, Porrozzi, \& Alves, 2002). Sin embargo, otros estudios han encontrado que el tiempo ontogenético puede generar una estabilidad numérica en la infracomunidad a través de bajas tasas de colonización y altos tiempos de residencia (Díaz \& George-Nascimento, 2002).

Las entidades parásitas Tetraphyllidea y Anilocra chromis son consideradas especies núcleo de la infracomunidad, con un efecto notable sobre la estructura comunitaria. Nuestros resultados apoyan la hipótesis de las especies núcleo que parasitan la población de hospederos antes que las especies raras y estocásticas y persisten durante la ontogenia (Vidal-Martínez, Aguirre-Macedo, VivasRodríguez, \& Moravec, 1998; Pérez de Olmo, 2008). La persistencia de las especies parásitas en la infracomunidad de C. cyanea a lo largo de las clases de talla reflejan un patrón anidado. Esto puede deberse a una acumulación de parásitos en el tiempo, que se mantiene para las especies transmitidas por vía trófica.

Los cambios obtenidos en los descriptores ecológicos antes y después de la talla de $6 \mathrm{~cm}$ en $C$. cyanea, podrían responder a un efecto acumulativo unido a los cambios en la dieta. Pastor \& Báez-Hidalgo (2003) determinaron que a los $6.5 \mathrm{~cm}$ de LT ocurre la maduración sexual de $C$. cyanea en ambos sexos; esta maduración es acompañada de un cambio radical en la dieta. Estos cambios en la dieta suplen las necesidades energéticas de la especie y consisten en la incorporación de un espectro y volumen mayor de presas. Los requerimientos energéticos de los hospederos podrían explicar la persistencia de las características cuantitativas, en tanto que la variación de la composición de las infracomunidades podría resultar de cambios en la oferta de presas.

Se concluye que el desarrollo ontogenético de Chromis cyanea constituye una variable importante en la formación de la infracomunidad parásita. Los taxa Tetraphyllidea y Anilocra chromis constituyen el núcleo de la infracomunidad parásita y la secuencia de aparición y persistencia de estas entidades durante el desarrollo ontogenético, apoyan la hipótesis de las especies núcleo. Los cambios en la infracomunidad a partir de la talla de $6 \mathrm{~cm}$ podrían ser el resultado de un efecto acumulativo unido a cambios en la dieta que aporta nuevos estadíos infectivos.

\section{AGRADECIMIENTOS}

Agradecemos a Víctor Vidal Martínez del CINVESTAV-IPN, Unidad Mérida, por sus oportunas e importantes recomendaciones. Igualmente agradecemos las opiniones de tres revisores anónimos que contribuyeron a mejorar una versión previa del manuscrito.

\section{RESUMEN}

Chromis cyanea es una de las especies marinas más comunes y abundantes de los arrecifes cubanos. En este estudio se cuantifica la infracomunidad de parásitos durante el desarrollo ontogenético de Chromis cyanea (Pisces: Pomacentridae) de la costa norte de La Habana, Cuba. Los objetivos son probar que las especies núcleo son responsables de la estructura en la infracomunidad y aparecen antes que las especies raras y estocásticas. Determinar si existe relación entre los descriptores ecológicos de la infracomunidad parásita con la longitud total. Se capturaron 278 
ejemplares de $C$. cyanea durante la estación de seca (Abril) del 2010. Se analizó un rango de tallas de 1.5 a $11.5 \mathrm{~cm}$ de largo total y se incluyeron ejemplares juveniles y adultos. Se recolectaron 2861 especímenes parásitos pertenecientes a 20 taxa: Crustacea (5), Nematoda (5), Trematoda (4), Cestoda (2), Monogenea (2), Turbellaria (1) y Ciliophora (1). Los taxa Tetraphyllidea y Anilocra chromis constituyen el núcleo de la infracomunidad parásita. La secuencia de aparición y la persistencia de estos taxa durante el desarrollo ontogenético apoyan la hipótesis de las especies núcleo. Los cambios en la infracomunidad, a partir de la talla de 6 $\mathrm{cm}$, podrían ser el resultado de un efecto acumulativo unido a cambios en la dieta que provocan la ingestión de nuevos estadíos infectivos. Se concluye que el desarrollo ontogenético de C. cyanea constituye una variable importante en la formación de la infracomunidad parásita.

Palabras clave: Chromis cyanea, infracomunidad, ontogenia, parásitos, peces marinos, especies núcleo, Cuba.

\section{REFERENCIAS}

Böhlke, J. E., \& Chaplin, C. G. (1968). Fishes of the Bahamas and adjacent tropical waters. Wynnewood, Pennsylvania: Livingston Publishing Co.

Bush, A. O., Lafferty, K. D., Lotz, J. M., \& Shostak, A. W. (1997). Parasitology meets ecology on its own terms: Margolis et al. revisited. Journal Parasitology, 83, 575-583.

Claro, R., Lindeman, K. C., \& Parenti, L. R. (2001). Ecology of the marine fishes of Cuba. Washington D.C.: Smithsonian Institution.

Cressey, R. (1991). Parasitic Copepods from the Gulf of Mexico and Caribbean Sea, III: Caligus. Washington, D. C.: Smithsonian Institution.

Díaz, F. \& George-Nascimento, M. (2002). Estabilidad temporal de las infracomunidades de parásitos en la borrachilla Scartichthys viridis (Valenciennes. 1836) (Pisces: Blenniidae) en la costa central de Chile. Revista Chilena de Historia Natural, 75, 641-649.

Dogiel, V. A., Petrushevski, G. K., \& Polyanski, Y. I. (1958). Parasitology of fishes. London, England: Oliver and Boyd.

Fernández, R. A. \& Corrada, R. I. (2009). Estudio taxonómico de la parasitofauna asociada a las principales familias de peces marinos de interés acuarístico (Informe final del proyecto de investigación del programa ramal científico-técnico Biodiversidad. DB015), Cuba: Acuario Nacional de Cuba.

Gonzáles, A. \& Vidal-Martínez, V. (2008). Las comunidades de helmintos del lenguado (Symphurus plagiusa) en la costa de Campeche, México. Revista Mexicana de Biodiversidad, 79, 159-173.
Hanski, I. (1982). Dynamics of regional distribution: the core and satellite species hypothesis. Oikos, 38, 210-221.

Holmes, J. C., \& Price, P. W. (1986). Communities of parasites. In J. Kikkawa, \& D. J. Anderson (Eds.), Community ecology: pattern and process (pp. 187213). United Kingdom: Blackwell, Oxford.

Iannacone, J. \& Alvariño, L. (2009). Metazoos parásitos de Mugil cephalus Linnaeus, 1758 (Perciformes: Mugilidae:) procedentes del Terminal Pesquero de Chorrillos, Lima, Perú. Neotropical Helminthology, 3, 15-28.

Iannacone, J., Morón, L., \& Guizado, S. (2010). Variación entre años de la fauna de parásitos metazoos de Sciaena deliciosa (Tschudi, 1846) (Perciformes: Sciaenidae) en Lima, Perú. Latin American Journal of Aquatic Research, 38, 218-226.

Jongman, C., Braak, T., \& Tongeren, O. V. (1995). Data analysis in community and landscape ecology. New York, USA: Cambridge University.

Johnson, M. W., Nelson, P. A., \& Dock, T. A. (2004). Structuring mechanisms of yellow perch (Perca flavescens) parasite communities: host age, diet, and local factors. Canadian Journal Zoology, 82, 1291-1301.

Kensley, B., \& Schotte, M. (1989). Guide to the marine isopod crustaceans of the Caribbean. Washington D.C., USA: Smithsonian Institution.

Kennedy, C. R. (1990). Helminth communities in freshwater fish: structured communities or stochastic assemblages? In G. W. Esch, A. O. Bush, \& J. M. Aho (Eds.), Parasite communities: Patterns and processes (pp. 131-156). London, England: Chapman \& Hall.

Labropoulou, M., Machias, A., Tsimenides, N., \& Eleftheriou, A. (1997). Feeding habits and ontogenetic diet shift of the striped red mullet, Mullus surmuletus Linnaeus, 1758. Fisheries Research, 31, 257-267.

Luque, J. L., Porrozzi, F., \& Alves, D. R. (2002). Community ecology of the metazoan parasites of the argentine goatfish, Mullus argentinae (Osteichthyies: Mullidae), from the coastal zone of the state of Rio de Janeiro, Brazil. Revista Brasileira de Parasitologia Veterinaria, 11, 33-38.

Luque, J. L., Mouillot, D., \& Poulin, R. (2004). Parasite biodiversity and its determinants in coastal marine teleost fishes of Brazil. Parasitology, 128, 671-682.

Luque, J. L., \& Poulin, R. (2007). Metazoan parasite species richness in Neotropical fishes: hotspots and the geography of biodiversity. Parasitology, 134, 865-878.

Magurran, A. E. (1988). Ecological diversity and its measurement. New Jersey, USA: Princenton University Press.

Morand, S., Rohde, K., \& Hayward, C. (2002). Order in ectoparasite communities of marine fish is explained 
by epidemiological processes. Parasitology, 124, 57-63.

Moravec, F., Vargas, V. J., Vivas, R. C., Gonsalez, S. D., Mendoza, E. F., Alvarez, R. S., \& Güemez, R. J. (1995). Helminth parasites of Epinephelus morio (Pisces: Serranidae). Folia Parasitology, 40, 55-63.

Muñoz, G., Grutter, A. S., \& Cribb, T. H. (2006). Endoparasite communities of five fish species (Labridae: Chelininae) from Lizard Island: how important is the ecology and phylogeny of the hosts. Parasitology, 132, 363-374.

Muñoz, G. \& Delorme, N. (2011). Variaciones temporales de las comunidades de parásitos en peces intermareales de Chile central: hospedadores residentes vs temporales. Revista de Biología Marina y Oceanografia, 46, 313-327.

Muñoz, G., \& Zamora, L. (2011). Ontogenetic variation in parasite infracommunities of the clingfish Sicyases sanguineus (Pisces: Gobiesocidae). Journal Parasitology, 97, 14-19.

Noga, E. J. (1996). Fish Disease. Diagnosis and treatment. Missouri, USA: Mosby.

Oliva, M. E., \& Luque, J. L. (2002). Metazoan parasite infracommunities in five sciaenids from the central Peruvian coast. Memorias, Instituto Oswaldo Cruz, 93, 175-180.

Pastor, L. G. \& Báez-Hidalgo, M. (2003). Características biológicas del Cromis azul, Chromis cyanea (Pisces: Pomacentridae) en la costa norte de la Habana, Cuba. Revista de Investigaciones Marinas, 24, 133-137.

Pérez de Olmo, A. (2008). Biodiversity and structure of parasite communities in Boops boops (Teleostei: Sparidae) from the western Mediterranean and off the north east Atlantic coasts of Spain (Doctoral dissertation). Universitat de Valencia, Spain.

Poulin, R., \& Valtonen, E. T. (2001). Nested assemblages resulting from host size variation: The case of endoparasite communities in fish hosts. International Journal Parasitology, 31, 1194-1204.

Rodríguez, L. \& George-Nascimento, M. (1996). La fauna de endoparásitos metazoos del bacalao de profundidad Dissostichu seleginoides Smitt, 1898 (Pisces: Nototheniidae) en Chile central: aspectos taxonómicos, ecológicos y zoogeográficos. Revista Chilena de Historia Natural, 69, 21-33.

Sánchez-Ramírez, C., \& Vidal-Martínez, V. (2002). Metazoan parasite infracommunities of Florida Pompano (Trachinotus carolinus) from the coast of the Yucatan Península, Mexico. Journal Parasitology, 88, 1087-1094.

Sturges, H. (1926). The choice of a class-interval. Journal American Statistic Associate, 21, 65-66.

Vidal-Martínez, V. M., \& Poulin, V. (2003). Spatial and temporal repeatability in parasite community structure of tropical fish hosts. Parasitology, 127, 387 -398.

Vidal-Martínez, V. M., Aguirre-Macedo, M. L., Tomás, S., David, G. S., \& Edgar, F. M. (2001). Atlas de Helmintos de peces cíclidos de México. México: Conabio, Cinvestav.

Vidal-Martínez, V. M., Aguirre-Macedo, M. L., VivasRodríguez, C. M., \& Moravec, F. (1998). Las comunidades de macroparásitos del mero Epinephelus morio (Pisces: Serranidae) en la Península de Yucatán, México. Proceedings of Gulf and Caribbean Fisheries Institute, 50, 764-779.

Yamaguti, S. (1963). Systema Helminthum. Monogenea and Aspidocotylea Vol. IV. New York and London: Wiley Interscience.

Zelmer, D. A., \& Arai, H. P. (2004). Development of nestedness: Host biology as a community process in parasite infracommunities of yellow perch Perca flavescens from Garner Lake, Alberta. Journal of Parasitology, 90, 435-436. 